ARTIFICIAL SATELLITES, Vol. 51, No. $3-2016$
DOI: 10.1515/arsa-2016-0008

\title{
INVESTIGATION OF THE ACCURACY OF GOOGLE EARTH ELEVATION DATA
}

\author{
Dr Khalid L.A. El-Ashmawy \\ Al-Matria Faculty of Engineering, Department of Civil Engineering, \\ Helwan University, Cairo, Egypt \\ khalid85_2002@yahoo.com
}

\begin{abstract}
Digital Elevation Models (DEMs) comprise valuable source of elevation data required for many engineering applications. Contour lines, slope - aspect maps are part of their many uses. Moreover, DEMs are used often in geographic information systems (GIS), and are the most common basis for digitally-produced relief maps. This paper proposes a method of generating DEM by using Google Earth elevation data which is easier and free. The case study consisted of three different small regions in the northern beach in Egypt. The accuracy of the Google earth derived elevation data are reported using root mean square error (RMSE), mean error (ME) and maximum absolute error (MAE). All these accuracy statistics were computed using the ground coordinates of 200 reference points for each region of the case study. The reference data was collected with total station survey. The results showed that the accuracies for the prepared DEMs are suitable for some certain engineering applications but inadequate to meet the standard required for fine/small scale DEM for very precise engineering study. The obtained accuracies for terrain with small height difference can be used for preparing large area cadastral, city planning, or land classification maps.

In general, Google Earth elevation data can be used only for investigation and preliminary studies with low cost. It is strongly concluded that the users of Google Earth have to test the accuracy of elevation data by comparing with reference data before using it.
\end{abstract}

Keywords: Google Earth; Elevation Data Accuracy; Digital Elevation Model; Surfer; Terrain Zonum

\section{INTRODUCTION}

Topographic data is important for many Civil Engineering applications such as construction of canal, drainage, dams, bridges, highways, etc.

One of the most important data in topographic information is elevation. The success of the project sometimes required highly accurate elevation data with sufficient detail. Currently, several methods are available for obtaining the terrain elevation data of a given topography. Some of the most common practices being the conventional or modern land survey methods, aerial photogrammetry, satellite photogrammetry, radar interfermometry, Lidar scanning, global positioning system (GPS) etc. Some of the global elevation data obtained using any of these methods are publically available. The public availability of elevation data has 
revolutionized the entire process of topographic data collection for engineering research and application.

Since its launch in June 2005, GoogleEarth ${ }^{\circledR}$ has enjoyed ever increasing popularity as the go-to application for map lovers, navigators and armchair explorers. Free for download and installation on every computer system - PC, Mac and Linux - Google has made GoogleEarth ${ }^{\circledR}$ a portable, within-grasp virtual globe one is free to explore at one's leisure (Google, 2015).

Google Earth is a virtual globe based on 3D maps and geographical information program. It facilitate mapping of the Earth by the superimposition of images obtained from satellite imagery, aerial photography and geographic information system (GIS) 3D globe. Google Earth uses digital elevation model (DEM) data collected by NASA's Shuttle Radar Topography Mission (SRTM) enabling 3D view of the whole earth. Google Earth also supports managing 3D Geospatial data through Keyhole Markup Language (KML). Google Earth is useful for many applications such as earth resource mapping, visualizing earth feature, 3-D renderings of structures, town planning, simulation of disaster event such as of earthquakes using the Google Earth model, to monitor traffic speeds and congestion etc.

Google Earth provides high-resolution elevation data using the virtual globe system, which started in June 2005 and used Shuttle Radar Topography Mission (SRTM) data for its elevation baseline. Google Earth ${ }^{\mathrm{TM}}$, $\mathrm{s}$ elevation data are at a resolution 5 to 20 times higher than available South African 1:50 000 Chief Directorate Surveys and Mapping (CDSM) datasets (Hoffmann and Winde, 2010). However, at some places the RMSE error of SRTM DEM is more than its specified accuracy of $\pm 16 \mathrm{~m}$ (Sharma et al., 2010). If the terrain is highly vegetated, slope steeply than accuracy may be further reduced. Although SRTM data underlie the Google Earth elevation data, it has undergone continuous refinement through successive addition of high resolution data from various sources as they become available. In view of the above facts it is imperative to carry out an accuracy assessment elevation data available with Google Earth.

This study presents a method to extract elevation data from Google Earth using online free web tool, to generate DEMs for the extracted data using Surfer Software (Golden Software, 2012) and to investigate the accuracy of Google Earth elevations using total station survey derived elevation data as a reference data.

\section{CASE STUDY}

Elevation data in this study were collected in three different small regions in north coast of Egypt. These regions are Region 1, Region 2 and Region 3 and lie in Dabaa, El-Alamain and Marsa Mattroh cities respectively. The study regions are uninhabited and desert and have elevation differences of 5,15 and 25m respectively as shown in Figure 1.

Each region of the case study has two GPS control points. The control point numbers, ground coordinates and standard errors are available.

For assessing the elevation accuracy of Google Earth, each region has 200 reference points. Determination of the ground coordinates of the reference points will be explained later. 


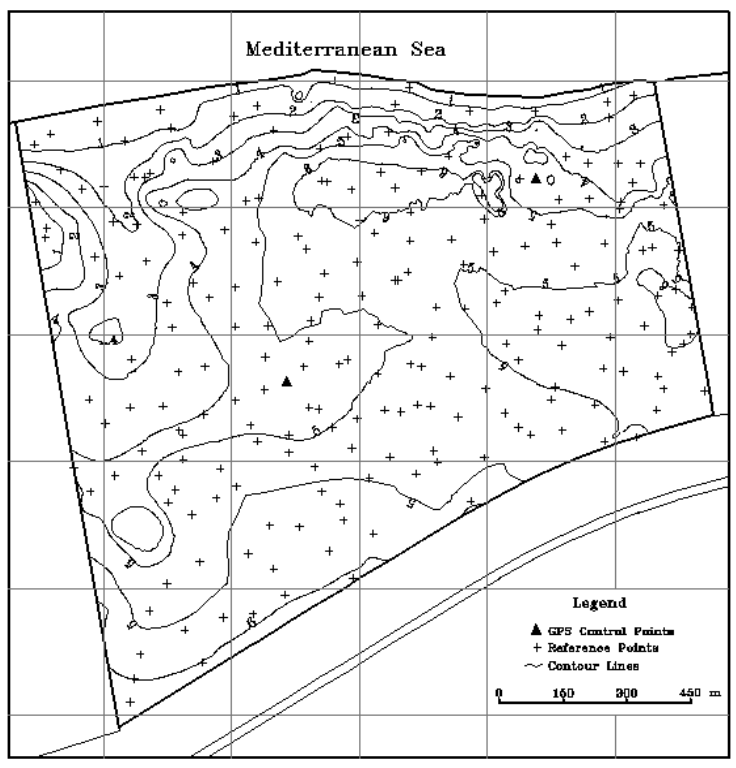

Fig. 1(a). Region 1

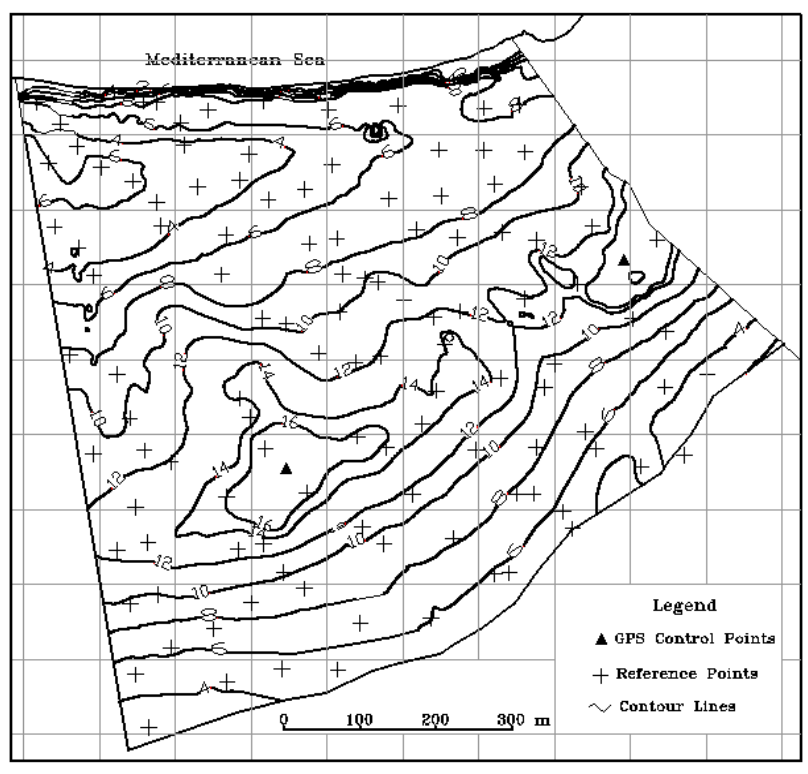

Fig. 1(b). Region 2

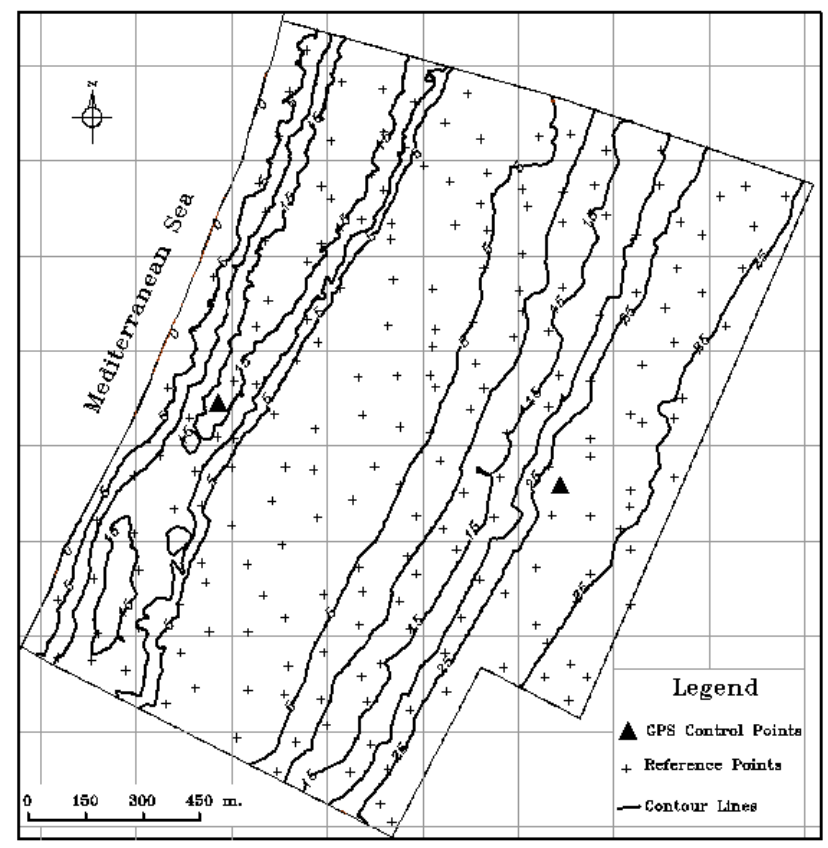

Fig. 1(c). Region 3

Fig. 1. Location of GPS control and Reference points in Study Regions 


\section{METHODOLOGY}

The followed methodology to achieve the objectives of this study is shown in Figure 2.

Total Station Survey for the determination of ground coordinates of two hundreds reference data points using the available two GPS control points.

Getting the ground coordinates of the reference data points

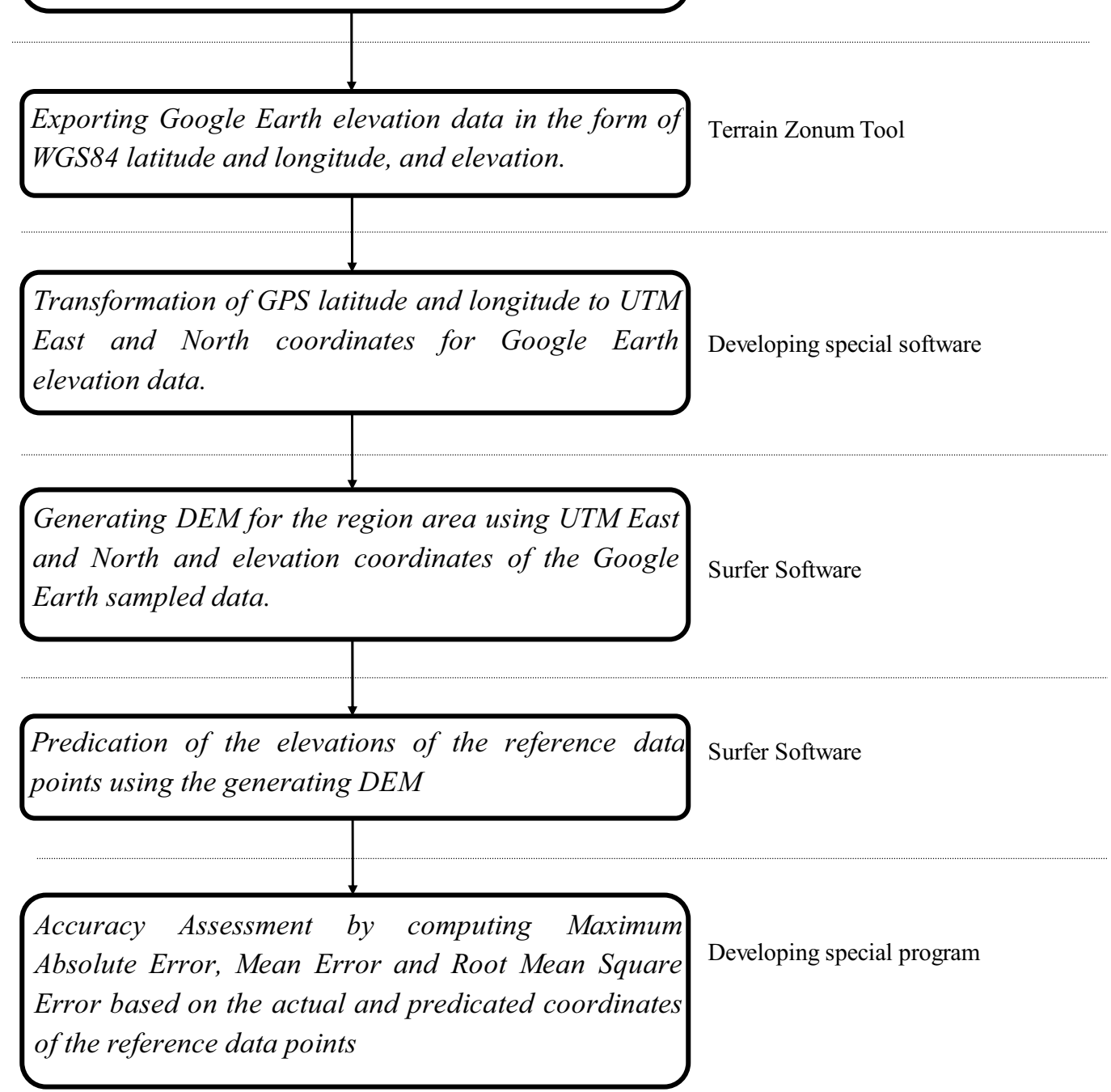

Fig. 2. The brief process of the methodology

\subsection{Getting the Reference data}

For each region of the case study, total station survey was used for getting the ground coordinates of two hundreds reference points.

Topcon GTS710 Total Station (Topcon, 2013) was used for data acquisition. This total station has the possibility to measure points up to 2400 meters on hard rock surface. Also, the total station has a large amount of memory to record all the data from the field. Besides this, the TS has software allows the surveyors to download the recorded data to a computer.

The collection of information on case study area was performed in two steps. The first step was started by accurate positioning of the instrument on the GPS ground control points, accurate leveling the instrument using a plate bubble and electronic level and measuring the 
instrument height to relate the location of the instrument to the known ground coordinates. The back sight target was positioned over a known ground control point and its height was measured to relate the target location to the ground coordinates. The back sight target was observed by the total station to orientate the survey.

The second step consisted of the observation of the desired points of the real natural terrain points, called side shots, by moving the prism with its pole on the ground. From these side shots, three-dimensional coordinates can be computed for the reference points. The two steps were repeated until surveying all reference points and recording the surveyed points for later processing.

All data obtained from the field was downloaded into computer using the capabilities of the available software with the total station. The coordinates of the interested points were exported to an ASCII file for post processing application.

\subsection{Exporting the elevation data of Google Earth}

Google Earth elevation data of each region were extracted using free source online tool

named Terrain Zonum (Terrain Zonum, 2015) via this website http://www.zonums.com/gmaps/terrain.php as following:

1. Going to http://www.zonums.com/gmaps/terrain.php?action=sample

a. Using the "Terrain" option to select the desired points in the selected region area using UNIFORM GRID. In this case, the number of rows and columns have to be specified. The maximum points should be not more than 5000 points i.e. 5000 elevation data. If detail elevation data is required, smaller area can be chosen and selecting bigger sampling points.

b. The "Extent" option is used to insert the maximum and minimum latitude and longitude of the region area. The used coordinate system is WGS 84 .

2. Clicking on GET ELEVATION button to sample the elevation points. The sampled points are described by latitude and longitude in decimal degrees and elevation in meters

3. After completing the 'sampling process', copy and paste the sampled data in Notepad and save as text file for further processing.

\subsection{Transformation of WGS84 latitude and longitude to UTM East and North coordinates}

A position on the Earth is given by the UTM zone number and the easting and northing coordinate pair in that zone. The point of origin of each UTM zone is the intersection of the equator and the zone's central meridian.

The transformation formulas are truncated version of Transverse Mercator: flattening series, which were described in Bomford, 1977. The WGS 84 spatial reference system describes Earth as an oblate spheroid along Equatorial axis of a $=6378173.00 \mathrm{~m}$, Polar axis of $\mathrm{b}=6356752.3142 \mathrm{~m}$ and an inverse flattening of $1 / \mathrm{f}=298.257223563$.

For a point of latitude $\varphi$ and of longitude $\lambda$, a reference meridian of longitude $\lambda_{0}$, the north $\mathrm{N}_{0}$ and east $\mathrm{E}_{0}$ coordinates of the origin point are $\mathrm{N}_{0}=0.0$ in the northern hemisphere or $\mathrm{N}_{0}=1000 \mathrm{~km}$ in the southern hemisphere and $\mathrm{E}_{0}=500 \mathrm{~km}$, and the scale factor $S_{0}=0.9996$, its UTM coordinates can be determined using the following formulas:

$$
E=E_{0}+S_{0} \cdot v \cdot\left[A+(1-T+C) \frac{A^{3}}{6}+\left(5-18 T+T^{2}+72 C-58 \bar{e}^{2}\right) \frac{A^{5}}{120}\right]
$$


Where:

$$
\begin{aligned}
N= & N_{0}+S_{0}\left[M-M_{0}+v \cdot \tan \phi\left(\frac{A^{2}}{2}+\left(5-T+9 C+4 C^{2}\right) \frac{A^{4}}{24}+\right.\right. \\
& \left.\left.\left(61-58 T+T^{2}+600 C-330 \bar{e}^{2}\right) \frac{A^{6}}{720}\right)\right]
\end{aligned}
$$

$$
\begin{aligned}
T= & \tan ^{2} \varphi \\
e^{2}= & \left(a^{2}-b^{2}\right) / a^{2} \\
\bar{e}^{2}= & \frac{e^{2}}{1-e^{2}} \\
C= & \bar{e}^{2} \cos ^{2} \varphi \\
A= & \left(\lambda-\lambda_{0}\right) \cos \varphi \\
M= & a \cdot\left[\left(1-\frac{e^{2}}{4}-\frac{3 e^{4}}{64}-\frac{5 e^{6}}{256}-\ldots .\right) \varphi-\left(\frac{3 e^{2}}{8}+\frac{3 e^{4}}{32}+\frac{45 e^{6}}{1024}+\ldots .\right) \sin 2 \varphi\right. \\
& \left.\quad+\left(\frac{15 e^{4}}{256}+\frac{45 e^{6}}{1024}+\ldots .\right) \sin 4 \varphi-\left(\frac{35 e^{6}}{3072}+\ldots .\right) \sin 6 \varphi+\ldots .\right]
\end{aligned}
$$

Based on the above mentioned formulation, menu based software is developed. The software reads the coordinates of points in the form of WGS84 latitude and longitude and transforms the coordinates to UTM E and $\mathrm{N}$ coordinates.

The developed software has been implemented using Visual $\mathrm{C}++$ Compiler V6.0 (Gregory, 1998) and designed to be flexible and portable to 32-bit Windows platforms. The software needs a minimum of 2 gigabytes of RAM memory and approximately 100 megabytes of disk space on the hard disk. It is released on DVD-ROM with a reference manual.

\subsection{Generating the DEM for each region Using the sampled data}

A DEM is a means or representing the shape of natural surfaces in digital form suitable for storage in a computer (Milne, 1987). To form a DEM, a detail survey is carried out in the area for which the DEM is required. Since the shape of natural surfaces varies in a random way, the network of points surveyed to represent the shape of the ground will usually form a random pattern consisting of horizontal coordinates with associated heights.

A DEM is usually formed from the field data using one of the following techniques.

- A square grid DEM is one in which data points are obtained at the nodes of a square grid. This model is formed by the computer interpolating the height of each grid node from the field data provided.

- A triangular grid DEM is one in which data points are interpolated at the corners of linked triangles which are positioned to give the best representation of the ground surface.

Representation of the DEM as a grid is quite common, as this format lends itself well to computer computations. This research is concerned solely with gridded DEMs. Here, the term DEM will refer to elevation represented by a regular gridded matrix. The quality of the DEM can vary greatly depending on the data source and the interpolation technique.

The DEM from the sampled points for each region was created using Surfer Software (Golden Software, 2012) to establish comparison consistency. Surfer is one of the well-known software packages used for contour and DEM generations. It is a product of Golden, Inc., Colorado, U.S.A. 
Surfer software reads the input data in the form of $\mathrm{X}, \mathrm{Y}, \mathrm{Z}$ ground coordinates of the irregularly and regularly spaced data points and creates a regularly gridded DTM for each region from which quasi-continuous surfaces could be produced. Grid density may be specified by either the number of grid lines along each side of the grid (X or $\mathrm{Y}$ ) or the distance between grid lines in $\mathrm{X}$ or $\mathrm{Y}$ data units along each side of the grid. Furthermore, the software allows the user to specify the interpolation method (griding method) to be used. There are twelve different interpolation methods available and the full description of these interpolation methods is explained by Golden Software, 2012. Out of the different methods of grid interpolation of Surfer software, Kriging method is recommended (El-Ashmawy and Azeez, 2005) and used in this research. After generating the grids file, DEM can be easily generated and viewed.

The results of using Surfer software are three grids files for the case study regions namely Region_1.grd, Region_2.grd and Region_3.grd. These files can be used for predicating the elevation of any point within the case study regions.

\subsection{Predication of the elevations of the reference data points using the generating DEM}

The Grid | Residuals command of the Surfer software can compute $Z$ value (elevation) of any point on a gridded surface. The operation can be performed as following:

- Creating an XYZ data file that contains the $\mathrm{X}$ and $\mathrm{Y}$ coordinates for the reference data points and values of " 0 " for the $Z$ coordinate.

- Selecting the grid and XYZ data files in the Open Grid dialog.

- Specifying, In the Grid Residuals dialog, the columns for X, Y, and Z and the column to which the residual values will be written in the worksheet.

- Using the Data | Transform command and multiply the reported residual values by negative one $(-1)$ to get the actual $\mathrm{Z}$ value of the surface at the points specified in the data file.

\subsection{Accuracy Assessment}

Once the elevations of the reference data points are known from the total station survey and Google Earth data, the elevation accuracy can be assessed in terms of Maximum Absolute Error (MAE), Mean Error (ME) and Root Mean Square Error (RMSE) and can be computed as following:

$$
\begin{aligned}
& \text { Absolute Error }=\mid \text { known elevation }- \text { predicated elevation } \mid \\
& \text { Mean Error }=\left(\sum_{i=1}^{n}(\text { known } \text { elevation }- \text { predicated } \text { elevation })_{i}\right) / n \\
& R M S E=\sqrt{\sum_{i=1}^{n}(\text { known } \text { elevation }- \text { predicated } \text { elevation })_{i}^{2} / n}
\end{aligned}
$$

Where $n$ is the number of reference data points.

\section{RESULTS AND DISCUSSION}

The obtained accuracy is the performance indicator key for the overall surveying operations. The accuracy is highly dependent on the surveying application, data, technique and the expected resulted production. The obtained accuracies of this research are shown in Table 1. 
Table 1. Reference data points elevation accuracy for the case study regions

\begin{tabular}{cccc}
\hline Method & Region_1 & Region_2 & Region_3 \\
\hline $\begin{array}{c}\text { Maximum Elevation } \\
\text { Difference (m) }\end{array}$ & 5 & 15 & 25 \\
\hline Maximum Absolute Error (m) & 3.72 & 6.39 & 8.78 \\
\hline Mean Error (m) & 0.51 & 1.13 & 1.52 \\
\hline RMSE (m) & 1.85 & 3.57 & 5.69 \\
\hline
\end{tabular}

From results of Table 1, it can be note that:

- Google Earth provides elevations with an accuracy of approximately of $1.85 \mathrm{~m}$ as a result of computing RMSE for height.

- Google Earth elevation data is more accurate in small height difference, or flat, area with $1.85 \mathrm{~m}$ RMSE value and error range less than $3.72 \mathrm{~m}$ and some findings less than $1 \mathrm{~m}$.

- Increasing the difference in height leads to decrease the obtained accuracy. For example increasing the height difference from 5 to $25 \mathrm{~m}$ increases the RMSE values from 1.85 to $5.69 \mathrm{~m}$.

The values of the obtained RMSE in Table 1 were compared with the permissible limits according to the specifications of ASPRS (American Society for Photogrammetry and Remote Sensing) (ASPRS, 1993) as tabulated in Table 2.

Table 2. ASPRS topographic elevation accuracy requirement for well-defined points

\begin{tabular}{cccc}
\hline \multirow{2}{*}{$\begin{array}{c}\text { Contour Interval } \\
\text { in Meters }\end{array}$} & \multicolumn{3}{c}{$\begin{array}{c}\text { ASPRS Limiting Root Mean Square Error in Meters } \\
\text { Spot or Digital Terrain Model Elevation Points }\end{array}$} \\
\cline { 2 - 4 } & Class I* & Class II & Class III \\
\hline 0.5 & 0.08 & 0.16 & 0.25 \\
1.0 & 0.17 & 0.33 & 0.5 \\
2.0 & 0.33 & 0.67 & 1.0 \\
4.0 & 0.67 & 1.33 & 2.0 \\
5.0 & 0.83 & 1.67 & 2.5 \\
\hline
\end{tabular}

* The maps are divided into three classes:

Class I holds the highest accuracies. Site plans for construction fit this category.

Class II has half the overall accuracy of Class I. Typical projects may include excavation, road grading, or disposal operations.

Class III has one third the accuracy or three times the allowable error of Class I maps. Large area cadastral, city planning, or land classification maps are typically in this category.

The following conclusions can be drawn:

- The obtained results for a terrain of $5 \mathrm{~m}$ height difference are suitable for generating contours map of contour interval $4.0 \mathrm{~m}$ or greater.

- The accuracies of the terrain of height difference greater than $5 \mathrm{~m}$ are unsuitable for generating contour map of any tabulated value of contour interval. 


\section{CONCLUSION}

Google Earth is an easy tool and is able to provide imagery and aerial view of the earth. The elevation data provided along with it may be used for preparing DEM.

The proposed method of this paper is simple for generating DEM from the extracted elevation data from Google Earth.

The obtained accuracies for the prepared DEMs are suitable for some engineering application but inadequate to meet the standard required for fine/small scale DEM for very precise engineering study. The accuracies for terrain with small height difference $(1.85 \mathrm{~m}$ RMSE) meet the vertical accuracy requirements of the ASPRS (1993) for the production of "Class III" contour maps. For this case, Google Earth can be used for preparing large area cadastral, city planning, or land classification maps. Furthermore, the results show that the accuracy requirements of ASPRS for generating contour maps cannot be met for the terrain of height difference greater than $5 \mathrm{~m}$ (RMSE $>2.5 \mathrm{~m})$.

However, Google Earth elevation data can be used for investigation and preliminary studies with low cost. It is strongly concluded that the users of Google Earth have to test the accuracy of elevation data by comparing with reference data before using it.

\section{REFERENCES}

ASPRS, American Society for Photogrammetry and Remote Sensing. (1993). ASPRS accuracy standards for large-scale maps. Bethesda (MD): ASPRS, USA.

Bomford, G. (1977), Geodesy. 3rd edition, Oxford Univ. Press, London, UK.

El-Ashmawy, K., and A.B. Azeez (2005). Generation of Mathematical Digital Terrain Model (DTM) Data for Testing DTM Generation Methodologies. Engineering Research Journal, Faculty of Engineering, University of Helwan, Egypt, Vol. 102, pp C 33 - C 49.

Golden Software (2012), Surfer Version 11: Reference Manual. Golden Software, Inc., Golden, Colorado, U.S.A.

Google (2015), About Google Earth: Understanding Google Earth imagery. Retrieved May 2, 2015 from http://earth.google.com/support/bin/answer.py?\&answer=176147.

Gregory, K. (1998), Special Edition Using Visual C++ 6. Que, USA.

Hoffmann, E. and F. Winde (2010). Generating high-resolution digital elevation models for wetland research using Google EarthTM imagery: an example from South Africa, Water SA. (Online), Vol. 36, pp. 53-68. (Accessed on 09/04/2014).

Milne, P.H. (1987), Computer Graphics for Surveying. E.\&F.N. Spon Ltd., London, UK.

Sharma, A., Tiwari, K.N. and P.B.S. Bhadoria (2010), Vertical Accuracy of Digital Elevation Model from Shuttle Radar Topographic Mission - A Case Study, Geocarto International, Vol. 25, No 4, pp 257-267.

Terrain Zonum (2015), Retrieved on October 12, 2015, from http://www.zonums.com/gmaps/terrain.php.

Topcon (2013), Retrieved on March 25, 2013 from http://www.topcon.co.jp/en/index.html

Received: 2016-04-29,

Reviewed: 2016-05-30 and 2016-06-14,

Accepted: 2016-09-15. 